\title{
Reflections on Preventive and Therapeutic \\ Strategies on Metastatic Germinoma in Abdomen: A Case Report and Literature Review
}

\section{Chenghai Zuo}

Third Military Medical University Southwest Hospital

\section{Yuhong Li}

Third Military Medical University Southwest Hospital

\section{Hua Feng}

Third Military Medical University Southwest Hospital

wenyan li ( $D$ lwy243@163.com )

First Affiliated Hospital of Third Military Medical University: Southwest Hospital

Jingyu Chen

Third Military Medical University Southwest Hospital

\section{Case report}

Keywords: germinoma, peritoneal metastasis, radiotherapy, chemotherapy

Posted Date: February 17th, 2021

DOl: https://doi.org/10.21203/rs.3.rs-229774/v1

License: (9) This work is licensed under a Creative Commons Attribution 4.0 International License. Read Full License 


\section{Abstract}

Background: We are introducing a case in which a patient of cranial germinoma developed peritoneal metastasis after ventriculo-peritoneal shunt and chemoradiotherapy. It would enlighten us to investigate the optimization of treatment on cranial germinoma when hydrocephalus exists to avoid peritoneal metastasis via shunt. Also, it required further investigations into the agent use in chemotherapy for metastatic germinoma.

Case presentation: The patient presented with severe obstructive hydrocephalus so that shunt was processed promptly. He then underwent chemoradiotherapy and tumor in pineal region diminished significantly. Unexpectedly, the patient began to complain of abdominal distention 4 months after the treatment and peritoneal metastasis of germinoma was confirmed. Finally, chemotherapy successfully treated the metastatic tumor as exhibited in the latest examinations.

Conclusions: This is a rare case we met amongst patients with cranial germinoma. It inspired us to reflect on the treatment optimization of cranial germinoma with hydrocephalus and standardization of agent use in metastatic germinoma.

\section{Background}

Among central nervous system (CNS) tumors, germ cell tumors (GCTs) are about $0.5 \%$ with germinoma as a major category (two thirds approximately). Over $90 \%$ of patients are confirmed before the age of 20 years[1]. Although they can develop in any location in CNS, pineal region and sellar region are the most predilection sites[2]. Symptoms and clinical signs of germinoma can be influenced by different factors. Endocrine deficiencies such as diabetes insipidus, precocious puberty and hypocortisolism occur when germinoma develops in sellar region. When extending to oppress optic chasm, it would lead to impaired visual fields. When germinoma occurs in pineal region, Parinaud's syndrome, obstructive hydrocephalus and midbrain incapability will be usually detected. Since germinoma is highly sensitive to radiotherapy and chemotherapy, reasonable remedy usually results in cure of the disease. However in this report, we are introducing a case in which peritoneal metastasis happened after the patient received ventriculoperitoneal (VP) shunt and concurrent chemoradiotherapy. This inspired us to discuss the proper timing of surgery and chemoradiotherapy, also the appropriate protocols of chemoradiotherapy for preventing metastasis and treating extra-neural germinoma.

\section{Case Presentation}

In 2018-03-09, this 23-year-old male presented with a headache in forehead. The distending headache was unbearable with vomiting, lasting 4-5min every time and relieving spontaneously. No other symptoms and positive signs were demonstrated. Cranial computed tomography (CT) exhibited the presence of occupation in the third ventricle in a hospital on 2018-03-11 (Fig. 1). He was primarily diagnosed with "germinoma" and "hydrocephalus", being admitted to our hospital on 2018-03-14. Blood 
sample demonstrated $\beta$-human chorionic gonadotropin ( $\beta$-HCG) to be $1.98 \mathrm{IU} / \mathrm{L}$ and alpha fetoprotein (AFP) to be $0.28 \mu \mathrm{g} / \mathrm{L}$ which were within normal range. Due to the urgency of the obstructive hydrocephalus, Ommaya reservoir insertion was operated on the left side on 2018-03-16 and VP shunt on the right side on 2018-03-21. Since the hydrocephalus was alleviated (Fig. 1), he underwent concurrent chemoradiotherapy from 2018-04-11 to 2018-05-24. Specifically, intensity modulated radiation therapy on brain: $32 \mathrm{~Gy}$ of clinical target volume and 34Gy of gross tumor volume; conformal radiation therapy on full-length spinal cord: 32Gy; a boost on primary location: 22Gy. Cranial MRI indicated that tumor in the third ventricle shrank to a great extent on 2018-05-24 (Fig. 1). Since then, he received chemotherapy for 11 times from 2018-08-20 to 2019-06-24. MRI recheck showed satisfied control of tumor progress with a diminishing size (Fig. 1).

However, the patient began to complain of abdominal distention on 2019-09-25, and was thus admitted to our hospital on 2019-10-12. CT illustrated giant lumps in abdomen and severe ascites on 2019-10-18 (Fig. 2). At the same time, ascites was collected for examination, showing Rivalta test $(+)$, lactate dehydrogenase to be $19139.9 \mathrm{U} / \mathrm{L}(<618 \mathrm{U} / \mathrm{L})$, protein level to be $42.0 \mathrm{~g} / \mathrm{L}$ and glucose to be $2.7 \mathrm{mmol} / \mathrm{L}$. Immunohistochemistry of the ascites revealed CK (+), SALL4 (+), WT-1 (-), CD68 (+) and oct 3/4 (-). Cytologic examination and H\&E staining of ascites were shown in Fig. 2. Therefore, the pathology was consistent with germinoma, indicating peritoneal metastasis. Following the evaluation of the patient by oncologists, he started to apply chemotherapy with etoposide and nedaplatin from 2019-11-04 to 202006-17. Tumor diminished tremendously and abdominal distention was alleviated during the chemotherapy, as exhibited by CT on 2019-12-18 and 2020-04-02 (Fig. 2). In the latest examinations, satisfied status in both brain and abdomen was evidenced (Fig. 2).

\section{Discussion And Conclusions}

In this report, we introduced the development and treatment of peritoneal metastasis of germinoma. VP shunt was first conducted due to the urgent condition of obstructive hydrocephalus. When the patient's condition fulfilled the requirements, standard chemoradiotherapy was processed and the tumor in brain diminished significantly. However, about 4 months after the chemoradiotherapy, germinoma was verified to metastasize to abdomen. He then received chemotherapy for over 6 months and abdominal germinoma was cured with no relapse detected 4 months since then.

As a rare tumor in CNS, germinoma showed a favorable prognosis with the 5-year survival rate higher than $85 \%$. This is attributed to the high sensitivity of the tumor to chemotherapy and radiotherapy[3]. In clinical trials from 1983-1993 conducted by German Cooperative, patients received mere craniospinal irradiation (CSI) with $36 \mathrm{~Gy}$, and 50Gy as a boost on primary sites). As a result, relapse was found in only one patient among 60 and the 5-year survival rate was over 93\%[4]. In comparison with CSI, Rogers et al. suggested whole-brain radiation led to similarly low incidence of spinal relapse[5]. Therefore, radiation alone could be effective, instead of the elevated possibility of long-term sequelae since it needs high dose and vast fields. As another approach, chemotherapy-only was investigated in clinical trials using regimens with carboplatin, bleomycin, etoposide and cyclophosphamide. However, this therapy is less 
effective than radiotherapy according to 5 -year survival rate and recurrence rate[6, 7$]$. In this context, concurrent chemoradiotherapy is believed to maximize the therapeutic effect of radiation and minimize the side effect of large dose and volume. Indeed, clinical trials in France and Japan demonstrated notably higher survival rate which could be over $98 \%[8,9]$. Surgical removal is not recommended because of the negligible benefits and additional risks[10], however, surgery for biopsy is acceptable when necessary[8].

This case inspired us about the opportunity of performing shunt and chemoradiotherapy. The patient complaint of severe headache and CT demonstrated remarkable obstructive hydrocephalus, so that VP shunt was initially operated in case of emergency. Following operation, concurrent chemoradiotherapy was performed as soon as his situation permitted. Although the treatment had never resulted in peritoneal metastasis in our hospital, there are cases presenting peritoneal metastasis via shunt. For example, Murray et al. introduced a case in which a 13-year-old female presented with hydrocephalus. VP shunt was placed, followed by craniospinal radiotherapy and the tumor was eliminated completely[11]. Although shunts are effective tools draining excessive craniospinal fluid (CSF) to abdomen, they create a latent way for peritoneal metastasis of intracranial tumors. Some people advocated the shunts with filters to lower the probability, nonetheless, the chance of shunt obstruction naturally increases[12]. As another means of CSF bypassing, endoscopic third ventriculostomy which creates a defect in floor of third ventricle communicates with interpeduncular cistern, precluding the risk of extra-neural metastasis of germinoma. However, when tumor developed on suprasellar region, the ventriculostomy will be dangerous. Furthermore, severe or even fatal complications such as bleeding and neural structure injury should be taken into considerations[13]. Although this patient exhibited notably sensitivity to chemotherapy and CSI, this could not exempt the chance of metastasis during the treatment. Therefore, timing of radiotherapy after VP shunt should be noticed because it may cause peritoneal metastasis if too late, or may be hazardous to wound healing and patients' general conditions if too early. As an alternative, performing radiation simultaneously with external drainage might be taken as a proper strategy, yet requiring clinical trials.

In treating abdominal germinoma in this patient, it was interesting that mere chemotherapy was sufficient. Remedy of etoposide plus platinum has long been verified effective in treating CNS germinoma, as evidenced in this case either. However, its therapeutic effects are generally inferior to radiotherapy and the reason might be the presence of blood-brain barrier (BBB)[14]. For this patient, chemotherapy illustrated excellent therapeutic effect on abdominal germinoma since agents would reach effective plasma concentration much easier in abdomen which lack BBB. Meanwhile, exemption of radiotherapy could also refrain patients from the risk of radiation complications such as enteritis[15].Therefore, chemotherapy with etoposide and platinum comes to the priority and additional choices such as dasatinib, temozolomide and GEMOX (gemcitabine and oxaliplatin) can also be applied[16-18]. To date, remedy of agents for treating metastatic germinoma lack a definite standard, and is probably dependent on factors such as age, serum markers, metastatic sites and sensitivity to platinum. For example, temozolomide showed negligible effect on patients of cisplatin-refractory metastatic germ cell tumor with AFP $>15 \mu \mathrm{g} / \mathrm{L}$ and elevation of $\beta-\mathrm{HCG}>2.2 \mathrm{mIU} / \mathrm{L}$, but it may not necessarily imply the effects on patients within other restrictions[17]. In short, intensive study on 
chemotherapy of metastatic germinoma is required for standardization of agent choice in diverse situations.

As concluded in this report, we introduced a patient with germinoma suffered from abdominal metastasis following standard chemoradiotherapy, inspiring us to reflect on the treatment of CNS germinoma with urgent hydrocephalus. This male received VP shunt when he was admitted to hospital due to the emergent hydrocephalus, followed by concurrent chemoradiotherapy and chemotherapy. However, giant germinoma metastasized to abdomen via shunt was confirmed 4 months later and then cured by chemotherapy for 6 cycles. This case inspired us to reflect on the treatment of CNS germinoma with hydrocephalus and suitable chemotherapy on metastatic germinoma.

\section{Abbreviations}

CNS

central nervous system

GCTs

germ cell tumors

VP

ventriculo-peritoneal

CT

computed tomography

$\beta-H C G$

$\beta$-human chorionic gonadotropin

AFP

alpha fetoprotein

CSI

craniospinal irradiation

CSF

craniospinal fluid

BBB

blood-brain barrier

GEMOX

gemcitabine and oxaliplatin

\section{Declarations}

Ethics approval and consent to participate: This report used images of the patient's imageological examinations and pathological examinations and did not include information of patient's privacy, so there should be no ethics concerns.

Consent for publication: A consent signed by the patient was provided. 
Availability of data and materials: The datasets used and/or analyzed during the current study are available from the corresponding author on reasonable request.

Competing interests: The authors declare that they have no competing interests.

Funding: National Natural Science Foundation Youth Project (81901213).

Authors' contributions: Chenghai Zuo: design of work; Yuhong Li: interpretation of data; Hua Feng: revision of manuscript; Wenyan Li*: draft of manuscript and design; Jingyu Chen*: revision and analysis. All authors approved the submitted version. They agreed both to be personally accountable for the author's own contributions and to ensure that questions related to the accuracy or integrity of any part of the work, even ones in which the author was not personally involved, are appropriately investigated, resolved, and the resolution documented in the literature.

\section{References}

1. Thakkar JP, Chew L, Villano JL. Primary CNS germ cell tumors: current epidemiology and update on treatment. Medical oncology (Northwood, London, England). 2013;30(2):496.

2. Kamoshima Y, Sawamura Y. Update on current standard treatments in central nervous system germ cell tumors. Curr Opin Neurol. 2010;23(6):571-5.

3. Osorio DS, Allen JC. Management of CNS germinoma. CNS oncology. 2015;4(4):273-9.

4. Bamberg M, Kortmann RD, Calaminus G, Becker G, Meisner C, Harms D, et al. Radiation therapy for intracranial germinoma: results of the German cooperative prospective trials MAKEI 83/86/89. J Clin Oncol. 1999;17(8):2585-92.

5. Rogers SJ, Mosleh-Shirazi MA, Saran FH. Radiotherapy of localised intracranial germinoma: time to sever historical ties? Lancet Oncol. 2005;6(7):509-19.

6. Kellie SJ, Boyce H, Dunkel IJ, Diez B, Rosenblum M, Brualdi L, et al. Primary chemotherapy for intracranial nongerminomatous germ cell tumors: results of the second international CNS germ cell study group protocol. J Clin Oncol. 2004;22(5):846-53.

7. Balmaceda C, Heller G, Rosenblum M, Diez B, Villablanca JG, Kellie S, et al. Chemotherapy without irradiation-a novel approach for newly diagnosed CNS germ cell tumors: results of an international cooperative trial. The First International Central Nervous System Germ Cell Tumor Study. J Clin Oncol. 1996;14(11):2908-15.

8. Finlay J, da Silva NS, Lavey R, Bouffet E, Kellie SJ, Shaw E, et al. The management of patients with primary central nervous system (CNS) germinoma: current controversies requiring resolution. Pediatr Blood Cancer. 2008;51(2):313-6.

9. Bouffet E, Baranzelli MC, Patte C, Portas M, Edan C, Chastagner P, et al. Combined treatment modality for intracranial germinomas: results of a multicentre SFOP experience. Société Française d'Oncologie Pédiatrique. Br J Cancer. 1999;79(7-8):1199-204. 
10. Souweidane MM, Krieger MD, Weiner HL, Finlay JL. Surgical management of primary central nervous system germ cell tumors: proceedings from the Second International Symposium on Central Nervous System Germ Cell Tumors. J Neurosurg Pediatr. 2010;6(2):125-30.

11. Murray MJ, Metayer LE, Mallucci CL, Hale JP, Nicholson JC, Kirollos RW, et al. Intra-abdominal metastasis of an intracranial germinoma via ventriculo-peritoneal shunt in a 13-year-old female. $\mathrm{Br} \mathrm{J}$ Neurosurg. 2011;25(6):747-9.

12. Halperin EC, Samulski T, Oakes WJ, Friedman HS. Fabrication and testing of a device capable of reducing the incidence of ventricular shunt promoted metastasis. J Neurooncol. 1996;27(1):39-46.

13. Bouras T, Sgouros S. Complications of endoscopic third ventriculostomy. World Neurosurg. 2013;79(2 Suppl):S22.e9-12.

14. Wang $D$, Wang $C$, Wang $L$, Chen $Y$. A comprehensive review in improving delivery of small-molecule chemotherapeutic agents overcoming the blood-brain/brain tumor barriers for glioblastoma treatment. Drug Deliv. 2019;26(1):551-65.

15. Loge L, Florescu C, Alves A, Menahem B. Radiation enteritis: Diagnostic and therapeutic issues. J Visc Surg. 2020;157(6):475-85.

16. Osorio DS, Finlay JL, Dhall G, Goldman S, Eisenstat D, Brown RJ. Feasibility of dasatinib in children and adolescents with new or recurrent central nervous system germinoma. Pediatr Blood Cancer. 2013;60(9):E100-2.

17. Varuni Kondagunta G, Bacik J, Schwartz L, Sheinfeld J, Bajorin D, Vuky J, et al. Phase II trial of temozolomide in patients with cisplatin-refractory germ cell tumors. Invest New Drugs. 2004;22(2):177-9.

18. Pectasides D, Pectasides M, Farmakis D, Aravantinos G, Nikolaou M, Koumpou M, et al. Gemcitabine and oxaliplatin (GEMOX) in patients with cisplatin-refractory germ cell tumors: a phase Il study. Ann Oncol. 2004;15(3):493-7.

\section{Figures}




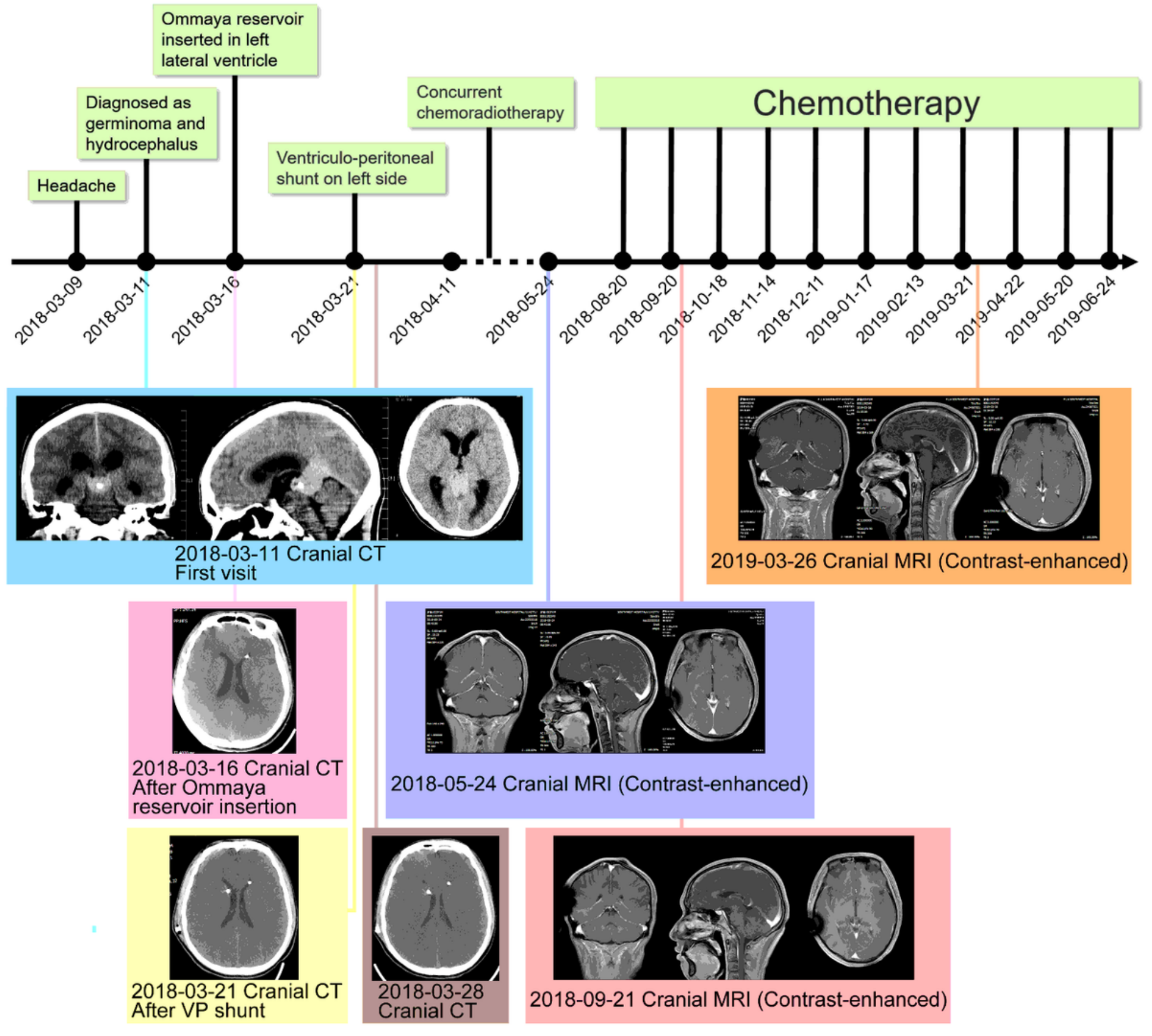

Figure 1

Timeline of the treatment of primary germinoma. Landmarks and dates of the entire treatment of the patient's primary germinoma with imageological studies. 


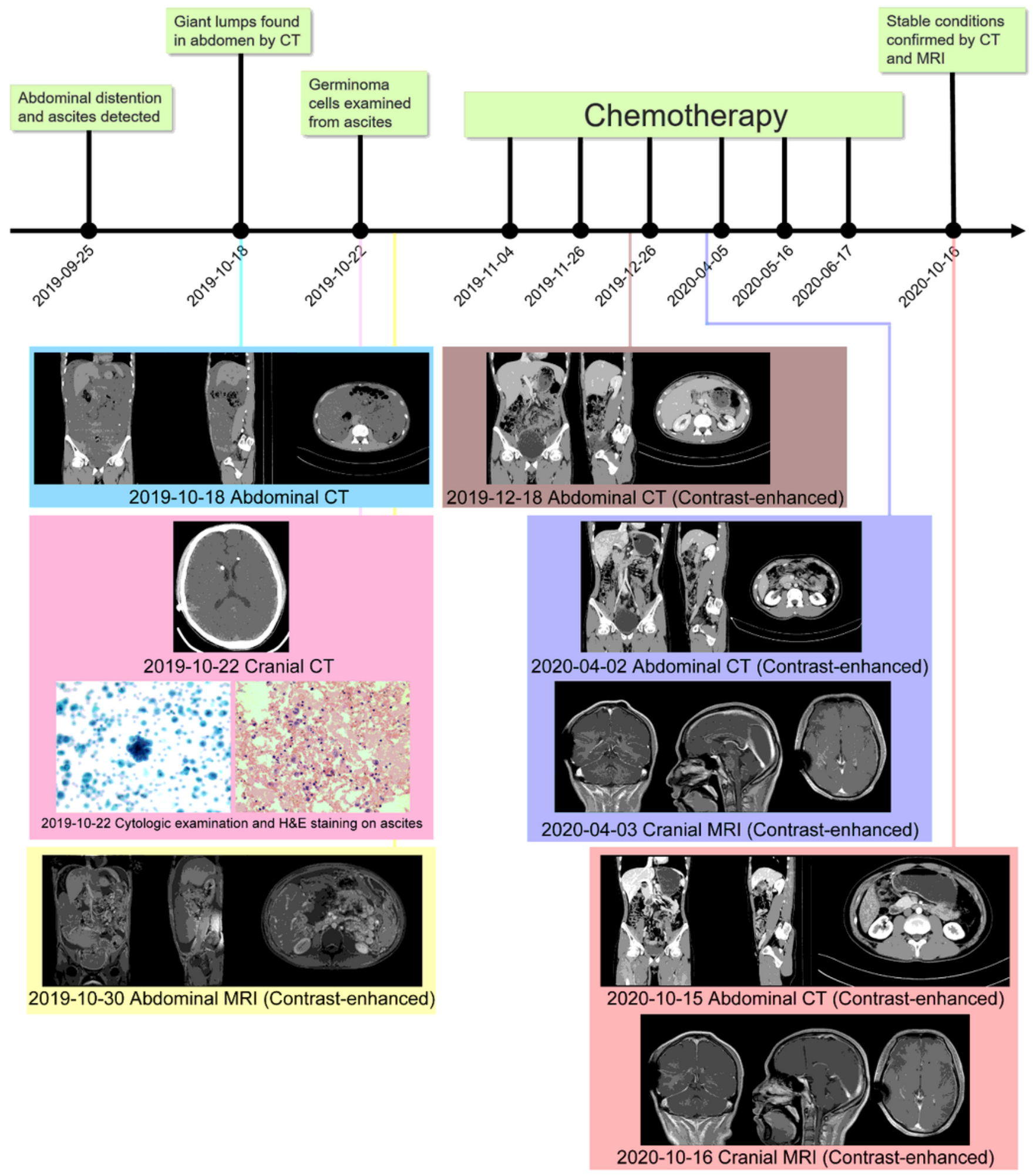

\section{Figure 2}

Timeline of the treatment of metastatic germinoma. Landmarks and dates from appearance of abdominal symptoms to the cure of germinoma in abdomen with cytologic staining and imageological studies. 